\title{
Bézier solutions of the wave equation
}

\author{
J.V. Beltran and J. Monterde \\ Dep. de Geometria i Topologia, Universitat de València, \\ Burjassot (València), Spain. beltranv@uv.es, monterde@uv.es
}

\begin{abstract}
We study polynomial solutions in the Bézier form of the wave equation in dimensions one and two. We explicitly determine which control points of the Bézier solution at two different times fix the solution.
\end{abstract}

\section{Introduction}

There are some papers dealing with Bézier or B-splines techniques applied to waves ([3], [2]), but, to our knowledge, there is not a study of the polynomial solutions in Bézier form of the wave equation.

The usual approach to the search of solutions to the wave equation is to state a Cauchy problem of the kind: To find a function verifying the wave equation with initial conditions the value of the function at time $t=0$ and the value at time $t=0$ of its partial derivative with respect to the time.

Along this paper the approach will be different. We shall try to find solutions of the wave equation with the value of the function at time $t=0$ as an initial condition, and with the value of the function in another time $t=t_{0}$ as a final condition.

For the 1D-wave equation this can be done using Bézier curves. Given an initial Bézier curve at time $t=0$ and another Bézier curve at another time, for the sake of simplicity, $t=1$, there is a unique Bézier solution of the 1D-wave solution verifying such initial and final conditions. Moreover, the control points of the Bézier solution are computed as the solution of a linear system from the control points of the initial and final Bézier curves.

This result can be useful to reproduce the motion of a string which evolves according to the wave equation. If snapshots of the string are taken at different times, then it is possible to interpolate the motion of the string by approximating first the string by a Bézier curve and finding then the Bézier solutions of the $1 \mathrm{D}$-wave equation according to our results.

For the 2D-wave equation the results are similar. Given an initial Bézier surface at time $t=0$ and another Bézier surface at time $t=1$, there is a unique Bézier solution of the 2D-wave solution verifying such initial and final conditions. The only difference is that the initial and final Bézier surfaces must verify some conditions. Such conditions can be expressed in terms of their control points and then some of the control points of the initial Bézier surface can be determined from the other control points. The same for the final Bézier surface. 
Usually, the wave equation is stated on functions. Nevertheless, here we are considering parametrized surfaces whose coordinate functions verify the wave equation. Due to the fact that we shall follow a Bézier approach, the amount of work needed is the same if we deal with parametrized surfaces or with functions.

Anyway, notice that the wave operator is not intrinsic to the surface in the same way that the usual Laplace operator is not intrinsic. In that case, the intrinsic operator is the Laplace-Beltrami operator. In spite of this, we shall study the non intrinsic wave operator as a first step towards the study of the corresponding intrinsic operator.

Moreover, if the first two coordinates are chosen appropriately, this is $P_{i j k}=$ $\left(\frac{i}{n}, \frac{j}{n}, c_{i j k}\right)$ for the $2 \mathrm{D}$-wave equation, then the third coordinate function of the Bézier surface can be understood as a function depending on the first two variables, and then, the wave equation has full sense.

\section{Bézier solutions of the 1D-wave equation}

If a string of uniform linear density is stretched to a uniform tension and if, in the equilibrium position, the string coincides with the $x$-axis, then when the string is disturbed slightly from its equilibrium position, the transverse displacement $u(x, t)$ satisfies an equation in which the second partial derivative of $u$ with respect to $x$ is proportional to the second partial derivative with respect to $t$,

$$
\frac{\partial^{2} u}{\partial x^{2}}=c^{2} \frac{\partial^{2} u}{\partial t^{2}}
$$

This is known as the one-dimensional wave equation. The usual approach is to state a Cauchy problem of the kind: To find a function $u(x, t)$ verifying Eq. 1 with initial conditions $u(x, 0)=u_{0}(x)$ and $\frac{\partial u}{\partial t}(x, 0)=v_{0}(x)$.

Along this section we shall take $c=1$ as the constant of proportionality. Then, the $1 \mathrm{D}$-wave equation can be written as

$$
\frac{\partial^{2} u}{\partial x^{2}}-\frac{\partial^{2} u}{\partial t^{2}}=0
$$

The only difference with the harmonic equation is a sign. So we can borrow the same study we did for the harmonic Bézier surfaces in [1] and [5]. Moreover, we shall look for solutions of Eq. 2 where not only for each $t_{0}$ the string $u\left(x, t_{0}\right)$ is a Bézier curve, but even the evolution along time of the string is of Bézier kind:

$$
u(x, t)=\sum_{i, j=0}^{m, n} B_{i}^{m}(x) B_{j}^{n}(t) P_{i j}, \quad x, t \in[0,1] .
$$

\subsection{The 1D-wave equation in terms of the control points}

A simple change of sign transforms the harmonic conditions (see [1], [4]) into the $1 \mathrm{D}$-wave conditions. 
Theorem 1. Given a control net in $\mathbb{R}^{3},\left\{P_{i j}\right\}_{i, j=0}^{m, n}$, the associated Bézier surface, $u:[0,1] \times[0,1] \rightarrow \mathbb{R}^{3}$, is a solution of the $1 D$-wave equation if and only if for any $i \in\{1, \ldots, m\}$ and $j \in\{1, \ldots, n\}$

$$
\begin{aligned}
& P_{i+2, j} a_{i m}+P_{i+1, j}\left(b_{i-1, m}-2 a_{i m}\right)+P_{i-1, j}\left(b_{i-1, m}-2 c_{i-2, m}\right)+P_{i-2, j} c_{i-2, m} \\
& -P_{i, j+2} a_{j n}-P_{i, j+1}\left(b_{j-1, n}-2 a_{j n}\right)-P_{i, j-1}\left(b_{j-1, n}-2 c_{j-2, n}\right)-P_{i, j-2} c_{j-2, n} \\
& +P_{i j}\left(\left(a_{i m}-2 b_{i-1, m}+c_{i-2, m}\right)-\left(a_{j n}-2 b_{j-1, n}+c_{j-2, n}\right)\right)=0,
\end{aligned}
$$

where, for $i \in\{0, \ldots, m-2\}$

$$
a_{i n}=(n-i)(n-i-1), \quad b_{i n}=2(i+1)(n-i-1), \quad c_{i n}=(i+1)(i+2),
$$

and $a_{i n}=b_{i n}=c_{i n}=0$ otherwise.

Note that the equations in the statement of the Theorem can be understood as generated by an adaptable mask.

The results obtained for harmonic Bézier surfaces indicate that given the first and last rows of control points, all the other control points are determined. The same is true for Bézier solutions of the 1D-wave equation.

For example, for $m=n=3$, given $P_{00}, P_{10}, P_{20}, P_{30}$ and $P_{03}, P_{13}, P_{23}, P_{33}$, the other control points are determined by

$$
\begin{aligned}
& P_{01}=\frac{1}{3}\left(4 P_{10}+2 P_{13}-2 P_{20}-P_{23}\right), \\
& P_{31}=\frac{1}{3}\left(-2 P_{10}-P_{13}+4 P_{20}+2 P_{23}\right), \\
& P_{02}=\frac{1}{3}\left(2 P_{10}+4 P_{13}-P_{20}-2 P_{23}\right), \\
& P_{32}=\frac{1}{3}\left(-P_{10}-2 P_{13}+2 P_{20}+4 P_{23}\right), \\
& P_{11}=\frac{1}{9}\left(-4 P_{00}-2 P_{03}+12 P_{10}+6 P_{13}-2 P_{30}-P_{33}\right), \\
& P_{12}=\frac{1}{9}\left(-2 P_{00}-4 P_{03}+6 P_{10}+12 P_{13}-P_{30}-2 P_{33}\right), \\
& P_{21}=\frac{1}{9}\left(-2 P_{00}-P_{03}+12 P_{20}+6 P_{23}-4 P_{30}-2 P_{33}\right), \\
& P_{22}=\frac{1}{9}\left(-P_{00}-2 P_{03}+6 P_{20}+12 P_{23}-2 P_{30}-4 P_{33}\right) .
\end{aligned}
$$

Let us recall that $\left\{P_{i 0}\right\}_{i=0}^{m}$ are the control points of the Bézier string at time $t=0$ and $\left\{P_{i n}\right\}_{i=0}^{m}$ are the control points of the Bézier string at time $t=1$. This gives us a non usual approach to the wave equation. Instead of fixing the initial state of the string and its first partial derivative with respect to the time we are fixing the initial and final states of the string.

The next figure shows the solution of the $1 \mathrm{D}$-wave equation as a two-dimensional surface. 


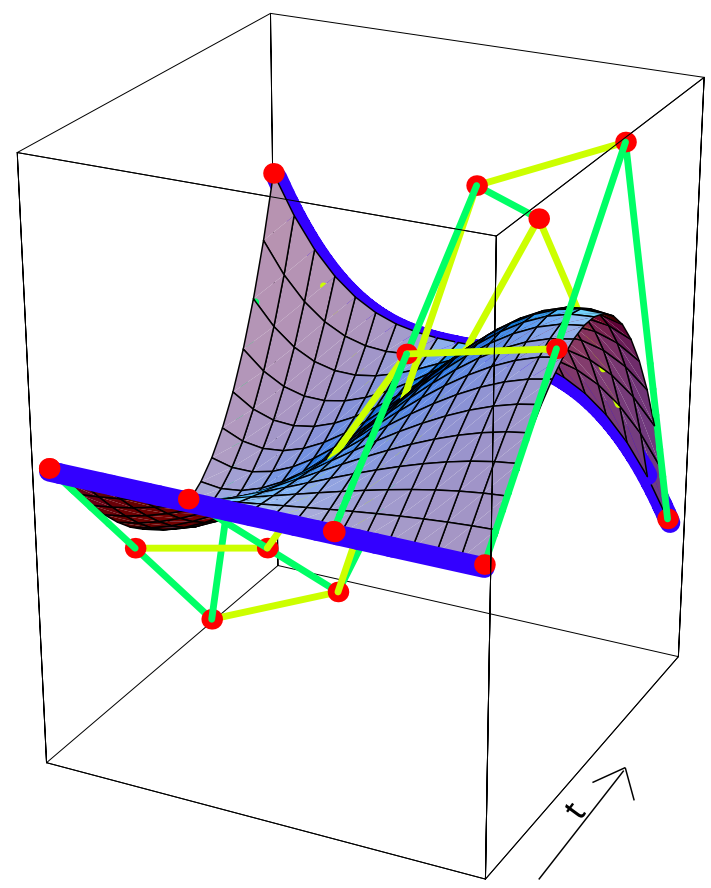

Figure I. A solution of the 1D-wave equation as a two-dimensional surface. The initial and final states are plotted thicker.

\subsection{Computing the solutions of the 1D-wave conditions}

We have seen that in low dimensions, the 1D-wave conditions imply that some of the control points can be expressed as linear combinations of the other control points. This is true for any dimension, i.e., the initial and final states fully determine the Bézier solution of the $1 \mathrm{D}$-wave equation. We prove that for a square net for simplicity.

Proposition 1. Let $u(x, t)=\sum_{i, j=0}^{n} B_{i}^{n}(x) B_{j}^{n}(t) P_{i j}$ be a Bézier solution of degree $n \geq 2$ of the $1 D$-wave equation with control net $\left\{P_{i j}\right\}_{i, j=0}^{n}$, then

1. If $n$ is odd, control points in the inner rows $\left\{P_{i j}\right\}_{i=1, j=0}^{n-1, n}$ are determined by the control points in the first and last rows, $\left\{P_{0 j}\right\}_{j=0}^{n}$ and $\left\{P_{n j}\right\}_{j=0}^{n}$.

2. If $n$ is even, control points in the inner rows $\left\{P_{i j}\right\}_{i=1, j=0}^{n-1, n}$ and also the corner control point $P_{n n}$ are determined by the control points in the first and last rows, $\left\{P_{0 j}\right\}_{j=0}^{n}$ and $\left\{P_{n j}\right\}_{j=0}^{n-1}$.

The analogous statement and proof for the harmonic case can be seen in [5]. We shall include here the proof for the 1D-wave case for the sake of completeness and because it gives some clues for what we will do later in the $2 \mathrm{D}$ case. 
Proof: Let us consider the case $n$ odd. Let us write the Bézier chart in the usual basis of polynomials

$$
u(x, t)=\sum_{i, j=0}^{n} \frac{A_{i j}}{i ! j !} x^{i} t^{j}
$$

where $A_{i j} \in \mathbb{R}^{3}$.

The 1D-wave equation $\left(\frac{\partial^{2}}{\partial x^{2}}-\frac{\partial^{2}}{\partial t^{2}}\right) u=0$ can be translated into a system of linear equations in terms of the coefficients $\left\{A_{i j}\right\}_{i, j=0}^{n}$

$$
A_{i+2, j}-A_{i, j+2}=0, \quad i, j=0, \ldots, n,
$$

but with the convention $A_{n+1, j}=A_{n+2, j}=A_{i, n+2}=A_{i, n+1}=0$.

This means that any coefficient $A_{i j}$ with $i>1$ can be related with $A_{i+2, j-2}$ and so on until the second subindex is 0 or 1 , or until the first subindex is greater than $n$. In this second case, $A_{i j}$ is directly 0 . Indeed, if $i+2 j>n$ then $A_{i, 2 j}=A_{i, 2 j+1}=0$, otherwise

$$
A_{i, 2 j}=A_{i+2 j, 0}, A_{i, 2 j+1}=A_{i+2 j, 1} .
$$

Note that the first and last rows of control points determine the starting and final curves $u(x, 0), u(x, 1), x \in[0,1]$. The first border curve is

$$
u(x, 0)=\sum_{i=0}^{n} A_{i 0} x^{i},
$$

and the second one is

$$
u(x, 1)=\sum_{i=0}^{n}\left(\sum_{j=0}^{n} A_{i j}\right) x^{i} .
$$

From Eq. 4 we can obtain coefficients $A_{i 0}$ for $i=0, \ldots, n$. Having in mind the expression of $u(x, t)$ in terms of Bernstein polynomials the formula of the change of basis from the basis of Bernstein polynomials to the usual basis says us that

$$
A_{i 0}=\left(\begin{array}{c}
n \\
i
\end{array}\right) \Delta^{i 0} P_{00}, \quad i=0,1, \ldots, n,
$$

where $\Delta^{i 0}$ is the incremental operator.

Thanks to Eq. 3, we can reduce Eq. 5

$$
\sum_{j=0}^{n} A_{i j}=\left(\begin{array}{c}
n \\
i
\end{array}\right) \Delta^{i 0} P_{0 n}, \quad i=0,1, \ldots, n,
$$

to just a system of linear equations involving the coefficients $A_{i 1}$.

Moreover, the matrix of coefficients of this system is triangular and with the unit in the diagonal entries. Therefore, the knowledge of the first and last rows of control points, implies the knowledge of the coefficients $A_{i 0}$ and $A_{i 1}$ and then, the knowledge of all the coefficients, i.e., of the whole solution $u(x, t)$, or equivalently, of the whole control net.

For the even case, the arguments are similar. 


\section{Bézier solutions of the 2D-wave equation}

If a thin elastic membrane of uniform areal density is stretched to a uniform tension and if, in the equilibrium position, the membrane coincides with the $x y$-plane, then the small transverse vibration $u(x, y, t)$ of the point $(x, y)$ of the membrane satisfies an equation in which the two dimensional Laplacian of $u$ is proportional to the second partial derivative with respect to $t$,

$$
\left(\frac{\partial^{2}}{\partial x^{2}}+\frac{\partial^{2}}{\partial y^{2}}\right) u=\Delta u=c^{2} \frac{\partial^{2} u}{\partial t^{2}} .
$$

This equation is called the two-dimensional wave equation.

As before, we shall assume that the constant of proportionality is $c=1$. Then, the $2 \mathrm{D}$-wave equation can be written as

$$
\left(\Delta-\frac{\partial^{2}}{\partial t^{2}}\right) u=0
$$

The operator $\square=\Delta-\frac{\partial^{2}}{\partial t^{2}}$ is sometimes called the D'Alembertian operator. It is the typical example of hyperbolic operator.

As in the previous section, we shall look for solutions of Eq. 6 where not only for each $t_{0}$ the membrane $u\left(x, y, t_{0}\right)$ is a Bézier surface, but even the evolution along time of the membrane is of Bézier kind:

$$
u(x, y, t)=\sum_{i, j, k=0}^{\ell, m, n} B_{i}^{\ell}(x) B_{j}^{m}(y) B_{k}^{n}(t) P_{i j k}, \quad x, y, t \in[0,1] .
$$

We shall call the set of control points $\left\{P_{i j k}\right\}_{i, j, k=0}^{\ell, m, n}$ the control web.

\subsection{The 2D-wave equation in terms of the control points}

Theorem 2. Given a control web in $\mathbb{R}^{3},\left\{P_{i j k}\right\}_{i, j, k=0}^{\ell, m, n}$, the associated Bézier membrane, $u:[0,1] \times[0,1] \times[0,1] \rightarrow \mathbb{R}^{3}$, is a solution of the 2D-wave equation if and only if for any $i \in\{1, \ldots, \ell\}, j \in\{1, \ldots, m\}$ and $k \in\{1, \ldots, n\}$

$$
\begin{aligned}
& a_{i \ell} \Delta^{200} P_{i j k}+b_{i-1, \ell} \Delta^{200} P_{i-1, j k}+c_{i-2, \ell} \Delta^{200} P_{i-2, j k} \\
& +a_{j m} \Delta^{020} P_{i j k}+b_{j-1, m} \Delta^{020} P_{i, j-1, k}+c_{j-2, m} \Delta^{020} P_{i, j-2, k} \\
& -a_{k n} \Delta^{002} P_{i j k}-b_{k-1, n} \Delta^{002} P_{i j, k-1}-c_{k-2, n} \Delta^{002} P_{i j, k-2}=0,
\end{aligned}
$$

where, for $i \in\{0, \ldots, \ell-2\}$

$$
a_{i n}=(n-i)(n-i-1), \quad b_{i n}=2(i+1)(n-i-1), \quad c_{i n}=(i+1)(i+2),
$$

and $a_{i n}=b_{i n}=c_{i n}=0$ otherwise.

The proof is analogous to that of harmonic Bézier surfaces (see [1]). To obtain a statement similar to that of Th. 1 all it lacks to do is to expand the incremental operators. 


\subsection{A tricubical example}

A study of Eqs. 8 for $\ell=m=n=3$ shows that given the border control points of the initial and final states $(2 \times 12)$ of the Bézier membrane, the other control points $(4+16+16+4)$ of the whole control web are determined. In the next Figure the independent border control points of the initial and final states are plotted thicker.
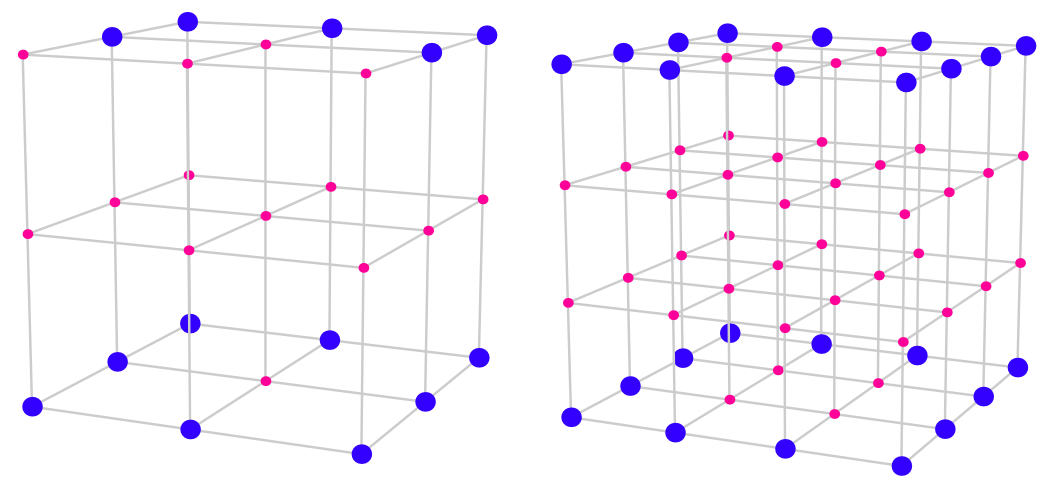

Figure II. Left $n=2$. Right $n=3$.

The Figure IV shows six states of the evolution of a solution for the 2D-wave equation with border control points (the black ones) in the initial and final states as follows in Figure III,

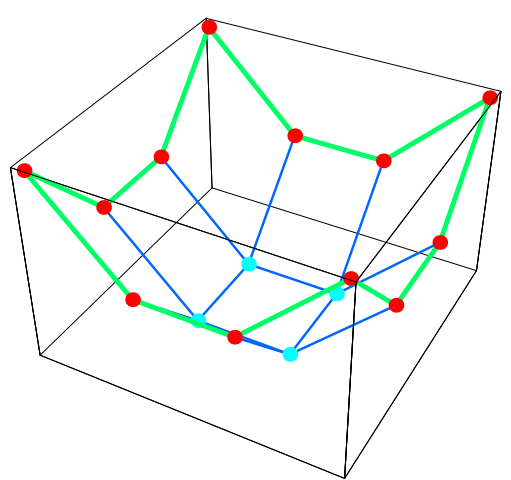

Initial state

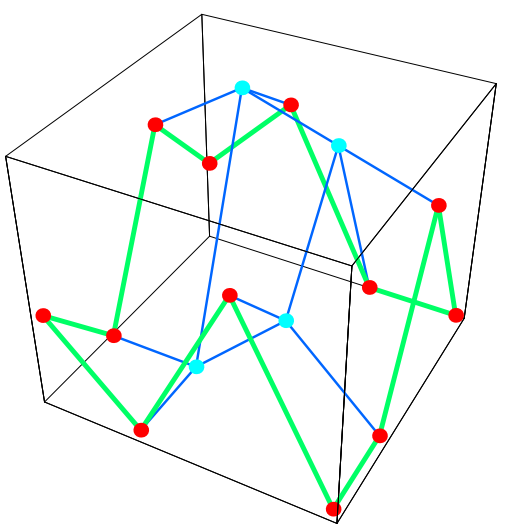

Final state

Figure III. 


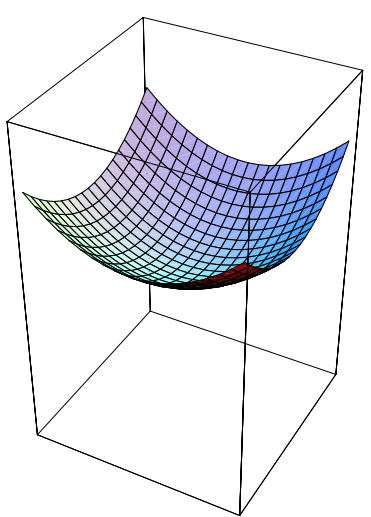

$\mathrm{t}=0.0$

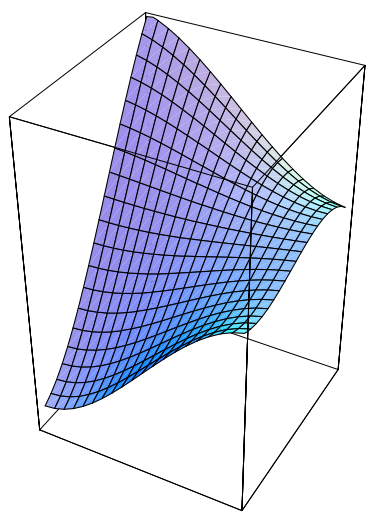

$\mathrm{t}=0.6$

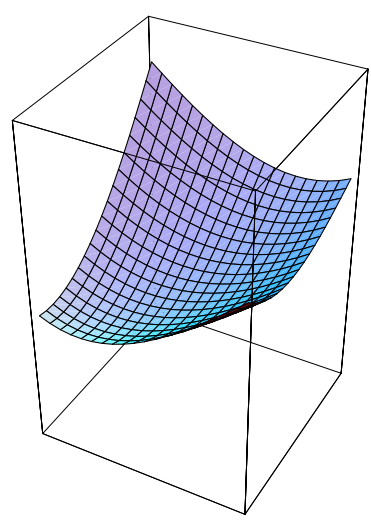

$t=0.2$

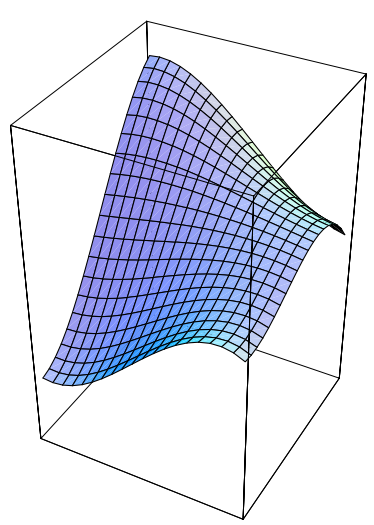

$\mathrm{t}=0.8$

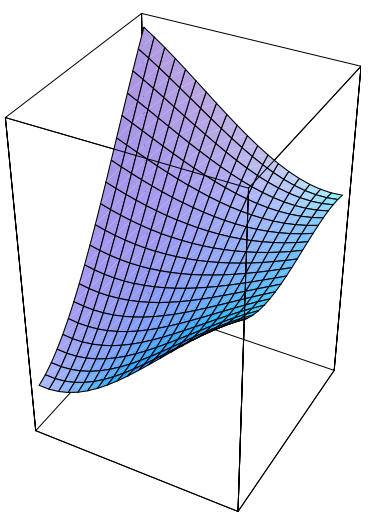

$\mathrm{t}=0.4$

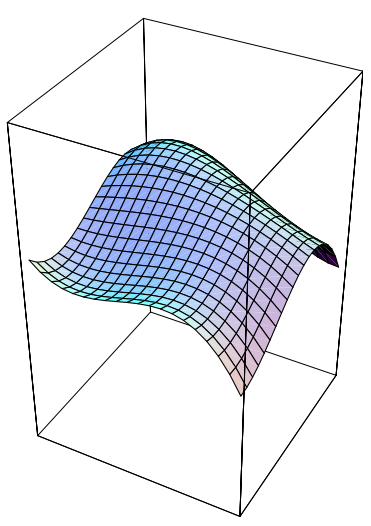

$\mathrm{t}=1.0$

Figure IV.

\subsection{Computing the solutions of the 2D-wave conditions}

To solve in general Eqs. (8) for degrees other than 2 and 3 is a difficult task even for a symbolic computation program. In order to do that, it is better to come back to the usual basis of polynomials.

Lemma 1. Let $f(x, y, t)=\sum_{i, j, k}^{n} \frac{a_{i, j, k}}{i ! j ! k !} x^{i} y^{j} t^{k}$ be a polynomial solution of degree $n \geq 2$ of the 2D-wave equation, then, all coefficients $\left\{a_{i j k}\right\}_{i, j=0, k=2}^{n}$ are totally determined by the coefficients $\left\{a_{i j 0}, a_{i j 1}\right\}_{i, j=0}^{n}$ and, moreover these two sets of coefficients verify if $n=2 p$ that

$$
\sum_{r=0}^{p}\left(\begin{array}{l}
p \\
r
\end{array}\right) a_{i+2 r, j+2(p-r), 1}=0 \quad, \quad \sum_{r=0}^{p+1}\left(\begin{array}{c}
p+1 \\
r
\end{array}\right) a_{i+2 r, j+2(p+1-r), 0}=0,
$$

and if $n=2 p-1$ that

$$
\sum_{r=0}^{p}\left(\begin{array}{l}
p \\
r
\end{array}\right) a_{i+2 r, j+2(p-r), 0}=0 \quad, \quad \sum_{r=0}^{p}\left(\begin{array}{l}
p \\
r
\end{array}\right) a_{i+2 r, j+2(p-r), 1}=0,
$$


for $i+j<n$ in both cases.

Proof: The 2D-wave condition $\square f=0$ can be translated into a system of linear equations in terms of the coefficients $\left\{a_{i, j, k}\right\}_{i, j, k=0}^{n}$

$$
a_{i+2, j, k}+a_{i, j+2, k}-a_{i, j, k+2}=0,
$$

for $i, j, k=0, \ldots, n$, but with the convention $a_{i j k}=0$ if any of the indexes is greater than $n$.

This means that any coefficient $a_{i j k}$ with $k>1$ can be written as $a_{i j k}=$ $a_{i+2, j, k-2}+a_{i, j+2, k-2}$ and so on until the last subindex is 0 or 1 , or until one of the first two subindexes is greater than $n$. Indeed,

$$
\begin{aligned}
a_{i, j, 2 k} & =\sum_{r=0}^{k}\left(\begin{array}{l}
k \\
r
\end{array}\right) a_{i+2 r, j+2(k-r), 0}, \\
a_{i, j, 2 k+1} & =\sum_{r=0}^{k}\left(\begin{array}{l}
k \\
r
\end{array}\right) a_{i+2 r, j+2(k-r), 1} .
\end{aligned}
$$

Note that using relations (12) we can solve all the Eqs. 11 in terms of $\left\{a_{i, j, 0}, a_{i, j, 1}\right\}$ for $k=0, \ldots, n-2$, but not for $k=n-1, n$. For these two values, Eqs. 11 are

$$
\begin{aligned}
a_{i+2, j, n-1}+a_{i, j+2, n-1} & =0, \\
a_{i+2, j, n}+a_{i, j+2, n} & =0,
\end{aligned}
$$

for $i, j=0, \ldots, n$, or, equivalently

$$
a_{i, j, n+1}=a_{i, j, n+2}=0,
$$

and with relations (12) we get the expressions (9) for $n$ even and (10) when $n$ is odd. Those are a set of relations between coefficients with last subindex 0 or 1. Moreover, it can be seen that if $i+j \geq n$ then the corresponding equations identically vanishes.

Now, let us translate this result in terms of the control web.

Proposition 2. Let $u(x, y, t)=\sum_{i, j, k=0}^{n} B_{i}^{n}(x) B_{j}^{n}(y) B_{k}^{n}(t) P_{i j k}$ be a Bézier solution of degree $n$ of the $2 D$-wave equation with $\left\{P_{i j k}\right\}_{i, j, k=0}^{n}$ as control web, then the control points in the inner levels $\left\{P_{i j k}\right\}_{i, j=0, k=1}^{n, n-1}$ are determined by the control points in the first and last rows, $\left\{P_{i j 0}\right\}_{i, j=0}^{n}$ and $\left\{P_{i j n}\right\}_{i, j=0}^{n}$. Moreover, there are also central control points of the initial and final states which are determined by the other control points.

Sketch of proof: Let us write the Bézier solution in the usual basis of polynomials

$$
u(x, y, t)=\sum_{i, j, k}^{n} A_{i j k} x^{i} y^{j} t^{k},
$$

where $A_{i j k} \in \mathbb{R}^{3}$. Coefficients with $k=0$ are determined by $u(x, y, 0)$, or, equivalently, by control points $\left\{P_{i j 0}\right\}_{i, j}^{n}$. Indeed, by the formula of the change of basis from the basis of Bernstein polynomials to the usual basis

$$
A_{i j 0}=\left(\begin{array}{c}
n \\
i
\end{array}\right)\left(\begin{array}{l}
n \\
j
\end{array}\right) \Delta^{i j 0} P_{000}
$$


where $\Delta^{i j 0}$ is the incremental operator.

Now, Eqs. (9) and (10) can be translated and solved in terms of the control points at time $t=0$.

The change $t \rightarrow 1-t$ in $u(x, y, t)$ allows to compute the analogous relations of the control points at time $t=1$. So, the same dependence scheme is valid for the initial and the final states.

Finally, as it happens for harmonic surfaces, the computation of the coefficients $A_{i j 1}$ from the control points of the initial and final states is reduced to a triangular linear system.

Once the first two levels of coefficients $A_{i j k}$ are known, then, by Lemma 1 , the solution $u(x, y, t)$ is completely known and, therefore, all its control points. This uniquely solves Eqs. (8) for $u(x, y, t)$ as in (7).

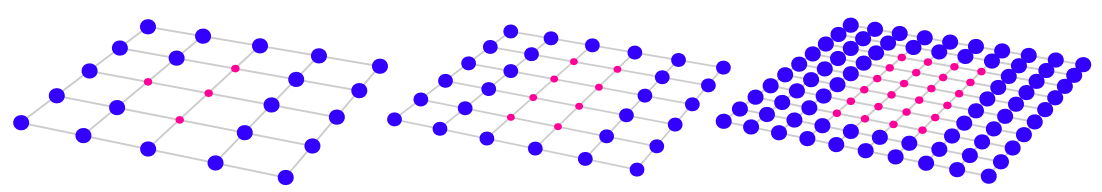

Figure V. Representation for $n=4$ (left), $n=5$ (center) and $n=9$ (right) of the independent control points (the bigger ones) in the initial and final states.

Compare with $n=2$ and $n=3$, Fig. II.

Note that for higher degrees, the number of control points near to the boundary increases. This means that we can fix not only the boundary of the initial and final states, but also, some of the partial derivatives of the initial and final states at the boundary.

\section{References}

1. C. Cosín, J. Monterde, Bézier surfaces of minimal area, Proceedings of the International Conference of Computational Science, ICCS'2002, Amsterdam, eds. Sloot, Kenneth Tan, Dongarra, Hoekstra, Lecture Notes in Computer Science, 2330, vol II, pages 72-81, Springer-Verlag, (2002).

2. B. Lang, The synthesis of waveforms using Bézier curves with control point modulation, The Second CEMS Research Student Conference.

3. A. T. Layton, M. van dePanne, A numerically efficient and stable algorithm for animating water waves, The visual Computer, 18, 41-53 (2002).

4. J. Monterde, Bézier surfaces of minimal area: The Dirichlet approach, to appear in CAGD, (2004).

5. J. Monterde, The Plateau-Bézier problem, in Mathematics of Surfaces X, M.J. Wilson and R.R. Martin (eds.), LNCS 2768, Springer-Verlag, 262-273, (2003). 